\title{
Estimating the Postmortem Interval (PMI) using Accumulated Degree-Days (ADD) in a temperate region of South Africa
}

J. Myburgh ${ }^{1}$, EN L’Abbé ${ }^{1}$, M Steyn ${ }^{1}$ and PJ Becker ${ }^{1,2}$

${ }^{1}$ Forensic Anthropology Research Centre, Department of Anatomy, School of Medicine, Faculty of Health Sciences, University of Pretoria, South Africa

${ }^{2}$ Biostatistics Unit, Medical Research Council, South Africa

Number of pages: 27

Number of figures: 3

Number of tables: 4

Corresponding author: Jolandie Myburgh, Department of Anatomy, Private Bag x323, Arcadia 0007, South Africa

Email: jolandie.myburgh@up.ac.za

Tel: +27 12 319-2263

Fax: +27 12 319-2240 


\title{
Estimating the Postmortem Interval (PMI) using Accumulated Degree-Days (ADD) in a temperate region of South Africa
}

\begin{abstract}
The validity of the method in which Total Body Score (TBS) and Accumulated Degree-Days (ADD) are used to estimate the postmortem interval (PMI) is examined. TBS and ADD were recorded for 232 days in northern South Africa, which has temperatures between 17 and $28{ }^{\circ} \mathrm{C}$ in summer and 6 and $20{ }^{\circ} \mathrm{C}$ in winter. Winter temperatures rarely go below $0{ }^{\circ} \mathrm{C}$. Thirty pig carcasses, which weighed between 38 and $91 \mathrm{~kg}$, were used. TBS was scored using the modified method of Megyesi et al. [1]. Temperature was acquired from an on site data logger and the weather station bureau; differences between these two sources were not statistically significant. Using Loglinear Random-effects Maximum Likelihood Regression, an $r^{2}$ value for ADD (0.6227) was produced and linear regression formulae to estimate PMI from ADD with a 95\% prediction interval were developed. The data of 16 additional pigs that were placed a year later were then used to validate the accuracy of this method. The actual PMI and ADD were compared to the estimated PMI and ADD produced by the developed formulae as well as the estimated PMIs within the $95 \%$ prediction interval. A validation of the study produced poor results as only one pig of 16 fell within the 95\% interval when using the formulae, showing that ADD has limited use in the prediction of PMI in a South African setting.
\end{abstract}

KEYWORDS: forensic anthropology, accumulated degree-days, postmortem interval, decomposition, total body score, pig models 


\section{Introduction}

South Africa's copious grasslands (veldt) are often used to dump a body or for the homeless to seek refuge and shelter. Since the majority of the country's population does not visit these areas frequently, a deceased person can easily reach an advanced stage of decomposition before discovery. Due to a large number of these cases in South Africa, research involving the estimation of the postmortem interval (PMI) is crucial for both forensic anthropologists and pathologists. While an accurate postmortem interval is needed to establish time since death and to exclude individuals who did not die within that time period [1], many of these methods have not been tested in geographical regions outside of the United States or Europe.

Researchers have described morphological changes that take place during decomposition in detail [2-6]. However, most of these descriptions are qualitative in nature; are based on personal opinions and experience; and are not applicable to all geographic and environmental conditions [1,7]. Additionally, many decomposition studies have been conducted in different seasons and climatic conditions using varying methodologies.

Since environment plays a large role in the rate of decomposition, the applicability of current PMI models need to be tested in a South African setting. In addition, postmortem interval estimates need to be created for specific geographic regions in the country. A model that is appropriate for comparison is Accumulated Degree-Days (ADD). ADD are heat-energy units that represent the accumulation of thermal energy that is needed for chemical and biological reactions to take place in soft tissue during decomposition [1]. For this reason, ADD represents chronological time and temperature combined [8]. Simmons and colleagues [8] explained this 
concept and stated that ADD measures the energy that is placed into a system as accumulated temperature over time and when an equal amount of thermal energy (ADD) is placed into a body or carcass, an equal amount of reaction (decomposition) is expected to take place. By making use of ADD, the effect of temperature in multiple regions is eliminated and comparisons between different studies can be made [8].

In 2005, Megyesi and colleagues proposed that Total Body Score (TBS) and ADD could be used to quantitatively estimate the postmortem interval [1]. This retrospective study made use of 68 forensic cases from a variety of environmental settings. Only cases with PMIs less than one year were used as little soft tissue was observed on remains after this period. From photographs and case reports, these authors observed and scored the state of decomposition. The scoring method divided decomposition into its four broad categories: fresh, early, late, and skeletal. All categories were further sub-divided into point-valued stages that described the general morphological appearance of the head and neck, trunk and limbs. The scores assigned to each region were combined to produce TBS. Temperature data were collected from a National Weather Service Station and were used to calculate ADD. The temperature data were composed of daily maximum and minimum air temperature averages. Statistical analyses of the TBS of each individual in the sample, plotted first against the known PMI and then the ADD, showed that decomposition increased rapidly then leveled off in a loglinear fashion. With linear regression analysis, Megyesi et al. [1] noted that approximately $80 \%$ of the variation in the decomposition process was attributed to ADD. Therefore, they suggested that decomposition should be modeled as being dependant on the accumulated temperature rather than just the elapsed time since death. 
Methods that use ADD may assist in the global application of time since death estimations in that knowledge of temperature can be used to adjust time scales for the postmortem interval. Many studies [8-10] have used ADD data to eliminate the effects of temperature and as a means to measure the effect other variables such as burial and submersion have on the rate of decomposition.

Three studies, Schiel [11], Parsons [12], and Suckling [13] tested the accuracy of the ADD/TBS method of Megyesi et al. [1] and developed new ADD formulae for three geographic regions in the USA. They made use of longitudinal, prospective studies to describe the decomposition process by observing the changes that take place with time and collecting multiple scores from a single body as it progresses through the stages of decomposition.

Schiel [11] used TBS scores from 10 pigs over two seasons (fall/winter in Iowa and spring/summer in Indiana) to develop estimates for ADD. The data, collected over a period of 6 months, were used to produce equations which were then compared to the quadratic equations developed by Megyesi et al. [1]. Comparisons of the mean error between the equations of the two studies revealed that the inaccuracy value (SE: 167.75) for the estimated ADD equations of Schiel [11] was less than that for the Megyesi et al. [1] equations (SE: 178.88). Furthermore, the decomposition curves obtained for the pigs were similar to the curves developed for the human sample. Approximately 73\% of the variation in decomposition was explained by ADD, which was similar to that observed by Megyesi and colleagues [1]. 
Parsons [12] used two pigs to study decomposition patterns in West Central Montana, USA. This study occurred during late summer (August 2008) and early fall (October 2008) and continued through the following spring (April 2009). Parsons [12] demonstrated that her PMI estimations were within the $80 \%$ confidence interval suggested by Megyesi et al. [1]. The patterns of decomposition observed in the study were used to suggest that ADD estimations became more accurate during the later stages of decay.

With a longitudinal study on human cadavers ( $\mathrm{n}=10)$, Suckling [13] validated Megyesi et al.'s [1] ADD prediction formulae. The formulae were shown to not be precise in predicting PMI for both early and advanced stages of decomposition (TBS > 22). In addition, she also suggested that scavengers contributed as much as temperature to decomposition rates.

The purpose of this study was to evaluate the method of Megyesi et al. [1] to estimate PMI from ADD and TBS in a South African climate. A longitudinal examination of these quantitative variables were conducted over a period of eight months (232 days) using a pig model. This period included both the colder winter and warmer summer temperatures. Data obtained were used to produce formulae to calculate the ADD which, in combination with temperature data from the national weather station, can ultimately be used to estimate the PMI. Also, data from independent pigs were used to test the accuracy and usability of these formulae.

\section{Materials and Methods}

A total of 46 pigs (Sus scrofa) were used in this study, 30 pigs to set up the standards and create region-specific formulae and 16 to validate these results. This study was conducted on the 
Miertjie Le Roux Experimental Farm, which belongs to the Faculty of Natural and Agricultural Sciences of the University of Pretoria. An enclosure was constructed in August 2008 by erecting a $50 \mathrm{~m}$ x $50 \mathrm{~m} 1.2 \mathrm{~m}$ high chicken wire fence on a half-hectare plot of veldt. The farm is situated on the central high veldt plateau of South Africa and the climate consists of warm, wind-free summer days (average daily temperatures of $23^{\circ} \mathrm{C}$ for December to February) and mild winters (average daily temperatures of $13^{\circ} \mathrm{C}$ for June to August), without snow, and with temperatures rarely below $0^{\circ} \mathrm{C}$. Rainfall mainly occurs during the summer months with hardly any winter showers (South African Weather Services). The vegetation in the area consists mostly of sour veldt grasslands or sour Bushveld [14].

Thirty pigs were placed on the farm at different times during the 232 day period (Table 1). Pig models were used in lieu of human cadavers and have widely been accepted as a means to evaluate the process of decomposition [11,15,16-20]. Pigs are considered to have similar decomposition patterns to humans due to their similarities in internal anatomy, intestinal flora, fat to muscle ratio and general hairlessness of the skin [15,21-23]. However, the timetables produced in pig studies should not be applied to human remains as the rates of decomposition may vary $[24,25]$. The carcasses were received from a local farmer and were placed in the enclosure no later than one day after death. According to the local farmer, the pigs' deaths had most likely been due to bacterial infections such as Listeria monocytogenes, Escherichia coli, and Clostridium perfringens. These diseases are common causes of death among pigs on commercial farms in South Africa. 
In order to minimize the influence of body size and weight on decomposition, piglets $(<38 \mathrm{~kg})$ and large pigs ( $>100 \mathrm{~kg}$ ) were excluded $[10,23,26]$. For the pigs used in this study, the farmer created two broad classification categories: 'porkers' (60 to $70 \mathrm{~kg}$ ) and 'baconers' (70 to $90 \mathrm{~kg}$ ). Prior to placement, the farmer weighed the pigs at his farm. Body weight ranged from $38 \mathrm{~kg}$ to $91 \mathrm{~kg}$ with an average weight of $71 \mathrm{~kg}$ (Table 1). After placement, the height, length, thoracic width, pelvic width and belly height were also measured. The mean values for these dimensions are as follows: height $(58.9 \mathrm{~cm})$, length $(130.3 \mathrm{~cm})$, width $(28.1 \mathrm{~cm})$, and belly height $(34.1 \mathrm{~cm})$. These dimensions were not included in further analyses, but may be assessed at a later stage to assess their influence on the decomposition process.

The state of decomposition was visually assessed and recorded three times a week for each pig until the tissues were desiccated. After this period, data were collected once a week. Data collection ended when the carcass had reached skeletonization (TBS score greater than 24).

The modified scoring method developed by Megyesi et al. [1], which is based on Galloway et al. [3], was used (Table 3). The scoring guidelines were applied to three regions of the body and were defined as follows:

- head and neck - from the snout to, but not including, the shoulder girdle;

- $\quad$ trunk - the shoulder girdle, thorax, abdomen and pelvic girdle; and

- limbs - where this appendage meets the body but excludes the shoulder and pelvic girdles. 
An example of a TBS is shown in Fig. 1. The flesh is caved in on the head and neck (score 7); post-bloat and black discolouration was noted on the trunk (score 5); and brownish shades of discoloration were noted on the limbs (score 4). The allotted point value for all three regions were added together to obtain a TBS of 16, which is used to represent the overall stage of decomposition for the carcass. The TBS values ranged from a minimum of 3 to a maximum of 35. These values represent different stages of decomposition.

Inter-class correlation was performed as a means to determine the repeatability of the proposed method of scoring the decomposition process. Inter-observer error was completed by an external individual scoring decomposition for the head and neck, trunk, limbs and the resulting TBS for the first ten pigs in the sample on the same day as the primary observer. If the coefficient of correlation is 1 , a perfect or complete correlation exists, values of between 0.75 and 0.99 indicate a high degree of correlation, 0.5 to 0.74 indicates a moderate degree of correlation while values of less than 0.49 indicate a low degree of correlation. If the correlation of the description scores is high (above 0.75 ) it shows that the degree of decomposition, as reflected by the TBS, can be consistently repeated [27]

In this study, average daily temperatures - the maximum and minimum air temperatures (in Celsius) - were obtained from a temperature data logger located on site (13 August 2008 - 15 January 2009) and from the South African Weather Bureau Station (16 January 2009 - 04 April 2009). The weather station is located approximately $23 \mathrm{~km}$ from the site. To calculate ADD, average daily temperatures were added from date of placement to skeletonization when data collection no longer took place. 
According to Dabbs [24], statistically significant differences might exist between recorded weather station and on site temperature data. In order to justify the combined use of the two above-mentioned datasets, an inter-class correlation was used to test the relationship between the data logger and weather station for a 175 day period (13 August to 15 January 2009). The intraclass correlation coefficient was 0.9 which indicates excellent inter-measure type agreement.

When using the Bland and Altman approach to measure agreement between the data logger and weather station temperature data, the limits of agreement for the two instruments were $-2.3^{\circ} \mathrm{C}$ to $2.1^{\circ} \mathrm{C}$. Therefore, accurate results can be achieved when the data logger data was as much as $2.1^{\circ} \mathrm{C}$ higher or $2.3^{\circ} \mathrm{C}$ lower than the weather station. The average daily difference in temperature for this period was $1.5^{\circ} \mathrm{C}$. The variation in temperature between the two instruments has not influenced the final result or its interpretations. Furthermore, the observed bias of $-0.113^{\circ} \mathrm{C}$ was considered negligible.

In order to assess the relationship between TBS and ADD, Random-effects Maximum Likelihood regression was used to model ADD. Because ADD values resulted in skewed distributions on the original scale, ADD was log transformed so as to be linearly related with TBS. These relationships were compared using their coefficients of determination $\left(\mathrm{r}^{2}\right)$. When multiplied by 100, the coefficients of determination express the percentage of the variation in $\log \mathrm{ADD}$ that can be explained by the variation in TBS. 
The models of ADD were reported on the original scale and predicted values of ADD were tabulated along with 95\% prediction intervals for all possible combinations of TBS.

In order to assess the accuracy of this method, the study was validated using data from 16 additional pigs that were placed at the Experimental farm in 2010 and 2011. The PMI and TBS of the pigs were recorded and the ADDs were calculated using the temperature data from the same weather station as in the original study. The TBS of each pig was entered into the formulae and the actual ADD was compared to the estimated ADD produced by the developed formulae. Also, the actual PMI was compared to the estimated PMI which was determined by counting the number of days it took for the actual ADD value to equal the estimated ADD value. The upper and lower limit PMIs were also calculated by counting the number of days it took for the ADDs to reach the estimated upper and lower limits of ADD.

Ethical approval for this study was obtained from the Faculty of Natural and Agricultural Sciences at the University of Pretoria, South Africa (ECO80617-026).

\section{Results}

To illustrate the progression of decomposition, TBS was plotted against ADD (Fig. 2). The pattern of decomposition followed an exponential shape. Regardless of season of placement of pigs, fresh and early decomposition (TBS $<15$ ) periods were relatively linear and were observed within 200 to 400 ADD after placement. In the later stages of decomposition, the pattern became highly variable (TBS values greater than 17) and the decomposition rate decreased to produce a 
plateau phase where decomposition remained relatively stable and unchanged for an extended period of time.

Different patterns of decomposition between winter and summer pigs were noted for the entire dataset (Fig. 2). For the early TBS scores, the rate of decomposition was similar in both samples. A difference between seasons was observed at a TBS of 17 and greater. After this stage, and with the same ADD values, pigs deposited during the summer period achieved higher TBS values than pigs deposited during winter. For example, at 1500 ADD, the TBS range for the summer period was 23 to 34 whereas the TBS range for winter pigs was 17 to 23 .

To use a linear model on the data, several assumptions needed to be met and include linearity, normal distribution, and heteroskedasticity. Due to the variable shape of the untransformed data, a Breasch - Pagan/ Cook - Weisberg test for heteroskedasticity was performed. The test for heteroskedasticity was not significant $(\mathrm{p}=0.9291)$ which supports the use of a linear model. Log transformation was used to linearize the data curve so as to permit the application of linear regression analysis (Fig. 3). Using the regression with log transformed data, a predictive equation was developed that can be used to calculate the estimated ADD for an unknown case:

$\mathrm{ADD}=10^{\text {(Coefficient*TBS + Intercept) }}$

$\mathrm{ADD}=10^{(0.073 * \mathrm{TBS}+1.135)}$

When TBS was regressed against $\log \mathrm{ADD}$, an $\mathrm{r}^{2}$ value of 0.623 was obtained. This means that $62 \%$ of the variability in decomposition as reflected by TBS is accounted for by ADD. 
This formulae estimate the possible number of ADD it would take for the pig to reach a specific stage of decomposition. Using all possible combinations of the predicted TBS values, ADD forecasts were produced from the above-mentioned equation $\left(\mathrm{ADD}=10^{\left(0.073^{*} \mathrm{TBS}+1.135\right)}\right)$ along with the upper and lower 95\% prediction intervals (Table 3).

For example, if a body with an unknown season of death was received and the TBS value was five, the estimated ADD would be 31.41 or between 24.98 and 39.48 . The result is an estimated PMI of approximately 4 to 7 days of average $6^{\circ} \mathrm{C}$ weather. Therefore, in order to transform the ADD into a PMI, the average of the daily temperatures needs to be obtained from the local weather station, and added together from the day of discovery until the actual ADD equals the estimated ADD. The number of days it took for these two values to equal will thus reflect the PMI.

However, the result from the validation study (Table 4) indicates that decomposition may be too variable to allow accurate PMI estimations. Of the 16 pigs, the PMI of only one (V16) pig fell within the lower limit of the 95\% prediction interval. The PMIs of 11 pigs were under estimated while the PMIs of 4 pigs were over estimated. Pigs V13 and V14 further demonstrate the influence of the variability of decomposition on the accuracy of this method. Both pigs had the same date of death and hence ADD/PMI values, however, V13 had a TBS of 3 while V14 had a TBS of 11. This resulted in drastically different estimated ADD values and thus estimated PMIs. Furthermore, V13 was underestimated while V14 was overestimated. 
The results for the inter-observer error indicated that this method is highly repeatable between observers. In general, TBS has the highest r-value of all the description scores and thus were correctly repeated $99.2 \%$ of the time. While the individual regions had lower r-values than that of the TBS, the r-values remained high ( 0.981 for head and neck, 0.985 for trunk and 0.990 for the limbs). The TBS scores were seldom different between the two observers; even though scores for one or more of the individual anatomical regions may have differed by one value. This resulted in TBS scores that were the same in certain instances, even though the scores for the various regions differed. However, the values produced by the Pearson's correlation test suggest that the descriptions for the different regions are clear enough so that individual scores and the TBS can be repeated in most instances.

\section{Discussion}

Forensic anthropologists in South Africa often receive human remains in an advanced stage of decomposition. Under these circumstances, the estimation of PMI can be important to help narrow down the number of potential victims. Because of a lack of information on the morphology and rate of decomposition in the climate in the northern region of South Africa, research involving methods to determine PMI from decomposed remains are important.

The use of ADD to estimate the postmortem interval has become a popular technique amongst forensic anthropologists, especially in North America [1,11,28]. Similar to other studies $[1,11,13]$, the rate of decomposition for the entire dataset was found to be curvilinear rather than linear. This implies that the initial rate of decomposition increased in a linear fashion but became more variable when TBS was greater than 17 . The reason for this variability is not entirely clear 
but it is expected that other factors start to play a larger role on the later decomposition processes. The decrease in the maggot mass size during the later stages of decomposition, for example, may have decreased the rate of decomposition [8]. Furthermore, the advanced decomposition phases occurred faster in the summer than in winter even at the same ADD. The summer period also displayed more variability. A possible explanation for this phenomenon of delayed decomposition in winter at the same ADD may be the fact that winters in the highveldt are often very dry, and it is not unusual that no rainfall occurs during this period. It is possible that some degree of desiccation / mummification may take place, which substantially delays the process of decomposition. This needs to be investigated in future studies.

Since the data was curvilinear, log transformation was employed to facilitate the Random-Effects Maximum Likelihood regression analyses. A large proportion (62\%) of the variation found in the TBS scores was explained with ADD. Therefore, morphological changes in the body are quantifiable. However, as can be seen from the decomposition pattern in Fig. 2 and discussed above, factors other than ADD play a role in the rate of decomposition. Differences in humidity, seasonal insect activity, possible bacterial infections prior to death and scavenger activity, for example, all have an influence and need to be further investigated [3,4,13,29,30].

These factors may all have contributed to the low levels of accuracy of this method when validated (Table 4). Even though formulae were developed for pigs in this environmental setting in particular, PMI estimates could not be accurately calculated when using data that conform to these specifics. This indicates that although TBS can be used to produce quantitative data, the process of decomposition is still too variable to make this method of much use when applying 
ADD. This is especially supported by the variability seen in pigs V13 and V14 which had the same PMIs, but dramatically different TBS values (3 and 11, respectively). Even if ADD is used to control for temperature, other factors resulted in differential rates of decomposition of pigs placed in the same environmental area. This shows that these other factors, e.g. scavenging, seasonality and bacterial infections, cannot be ignored when trying to estimate the PMI [13]. However, TBS is a good quantitative indicator of the stages of decomposition and the rate of change from one TBS value to another can be used to show the differences in the rate of decomposition between specimens. Future research in the South African setting may aim at developing separate predictive formulae for bodies deposited in summer and winter respectively, but the practical application of this may be difficult.

In conclusion, this study used a large sample of pigs to investigate the usability of TBS and ADD to predict the PMI in a temperate region of South Africa. On validation it was found that the process of decomposition is too variable to make this method of much use in this area, and it seems that especially seasonality played a major role in this part of the world.

\section{Acknowledgments}

This research was funded by the National Research Foundation of South Africa (NRF). Any opinions, findings and conclusions or recommendations expressed in the material are those of the authors and therefore the NRF do not accept any liability in regard thereto.

We would like to thank Natalie Keough for her valuable contributions to this study, Thomas van Deventer for providing the pigs and the NRF (National Research Foundation of South Africa) and Navkom (University of Pretoria) for funding. The South African Weather Services supplied 
the temperature data. Roelf Coertzen’s help at the Miertjie Le Roux Farm was invaluable, and we are grateful towards the Faculty of Natural and Agricultural Sciences for permission to use their facilities.

\section{References}

1. M.S. Megyesi, S.P. Nawrocki, N.H. Haskell, Using accumulated degree days to estimate the postmortem interval from decomposed human remains, J. Forensic Sci. 50 (2005) 618-26.

2. H. Reed, A study of dog carcass communities in Tennessee, with special reference to the insects, Am. Mid. Nat. 59 (1958) 213-45.

3. A. Galloway, W.H. Birkby, A.M. Jones, T.E Henry, B.O. Parks, Decay rates of human remains in an arid environment, J. Forensic Sci. 34 (1989) 607-16.

4. W.M. Bass, Outdoor decomposition rates in Tennessee, in: H. Haglund, M. Sorg, (Eds), Forensic taphonomy, CRC Press, Boca Raton, 1997, pp. 181-6.

5. M.A. Clark, M.B. Worrell, J.E. Pless, Postmortem changes in soft tissues, in: H. Haglund, M. Sorg, (Eds.), Forensic taphonomy, CRC Press, Boca Raton, 1997, pp. 151-64.

6. A. Galloway, The process of decomposition: a model from the Arizona-Sonoran desert, in: H. Haglund, M. Sorg, (Eds.), Forensic taphonomy, CRC Press, Boca Raton, 1997, pp. 13950.

7. W.D. Haglund, M.H. Sorg, Method and theory of forensic taphonomy research, in: H. Haglund, M. Sorg, (Eds.), Forensic taphonomy, CRC Press, Boca Raton, 1997, pp. 13-26.

8. T. Simmons, R.E. Adlam, C. Moffat, Debugging decomposition data - Comparative taphonomic studies and the influence of insects and carcass size on decomposition rate, J. Forensic Sci. 55 (2010) 8-13. 
9. V. Heaton, A. Lagden, C. Moffatt, T. Simmons, Predicting the postmortem submersion interval for human remains recovered from U.K. waterways, J. Forensic Sci. 55 (2010) 302307.

10. P. Cross, T. Simmons, The influence of penetrative trauma on the rate of decomposition, J. Forensic Sci. 55 (2010) 295-301.

11. M. Schiel, Using accumulate degree days for estimating the postmortem interval: A reevaluation of Megyesi's regression formulae [MSc thesis], University of Indianapolis, Indiana, 2008.

12. H.R. Parsons, The postmortem interval: A systematic study of pig decomposition in West Central Montana [MSc thesis], University of Montana, Montana, 2009.

13. J.K. Suckling, A longitudinal study on the outdoor human decomposition sequence in Central Texas [MSc thesis], Texas State University, Texas, 2011.

14. J.P.H. Acocks, Veld types of South Africa, third ed., Memoirs of the Botanical Survey of South Africa 57 (1988)1-146.

15. N.M. Reeves, Taphonomic effects of vulture scavenging, J. Forensic Sci. 54 (2009) 523-8.

16. J.A. Payne, A summer carrion study of the baby pig Sus scrofa Linnaeus. Ecology 46 (1965) 592-602.

17. T.T. Terneny, Estimation of time since death in humans using mature pigs [MSc thesis], University of Montana, Montana, 1997.

18. O.A. Shalaby, L.M. DeCarvalho, M.L. Goff, Comparison of patterns of decomposition in a hanging carcass and a carcass in contact with soil in Xerophytic habitat on the island of Oahu, Hawaii, J. Forensic Sci. 45 (2000)1267-73. 
19. A.W. Bunch, The impact of cold climate on the decomposition process, J. For. Identification. 59 (2009) 26-44.

20. C.A. Callahan, Vegetation colonization of experimental grave sites in Central Texas, [MSc thesis]. Texas State University, Texas, 2009.

21. M.L. Goff, Estimation of the postmortem interval using Arthropod development and successional patterns, Forensic Sci. Rev. 81 (1993) 81-94.

22. J.H. Byrd, J.L. Castner, Insects of forensic importance, in: J.H. Byrd, J.L. Castner, (Eds.), Forensic entomology: the utility of arthropods in legal investigations, CRC Press, Boca Raton, 2001, pp. 43-80.

23. C.M. Pakosh, T.L. Rogers, Soft tissue decomposition of submerged, dismembered pig limbs enclosed in plastic bags, J. Forensic Sci. 54 (2009) 1223-8.

24. R.P. Lane, An investigation into Blowfly (Diptera: Calliphoridae) sucession on corpses. J Nat. Hist. 9(1975): 581-588.

25. E.P. Catts, M. L. Goff, Forensic entomology in criminal investigations. Annu Rev. Entomol. 37(1992): 253-272.

26. G.R. Dabbs, Caution! All data are not created equal: The hazards of using National Weather Service data for calculating accumulated degree days, Forensic Sci. Int. 292 (2010) 49-52.

27. J.C. Allan, About correlation, in: Learning about statistics, Macmillan South Africa (publishers) (PTY) Ltd. Johannesburg, South Africa, 1982, pp. 122-140.

28. A.A. Vass, W.M. Bass, J.D. Wolt, J.E. Foss, J.T. Ammons, Time since death determinations of human cadavers using soil solution, J. Forensic Sci. 37 (1992) 1236-53. 
29. R.W. Mann, W.M. Bass, L. Meadows, Time since death and decomposition of the human body: variables and observations in case and experimental field studies, J. Forensic Sci. 35 (1990) 103-11.

30. C.P. Campobasso, G. Di Vella, F. Introna, Factors affecting decomposition and Diptera colonization, Forensic Sci. Int. 2 (2001) 18-27. 


\section{Figures}

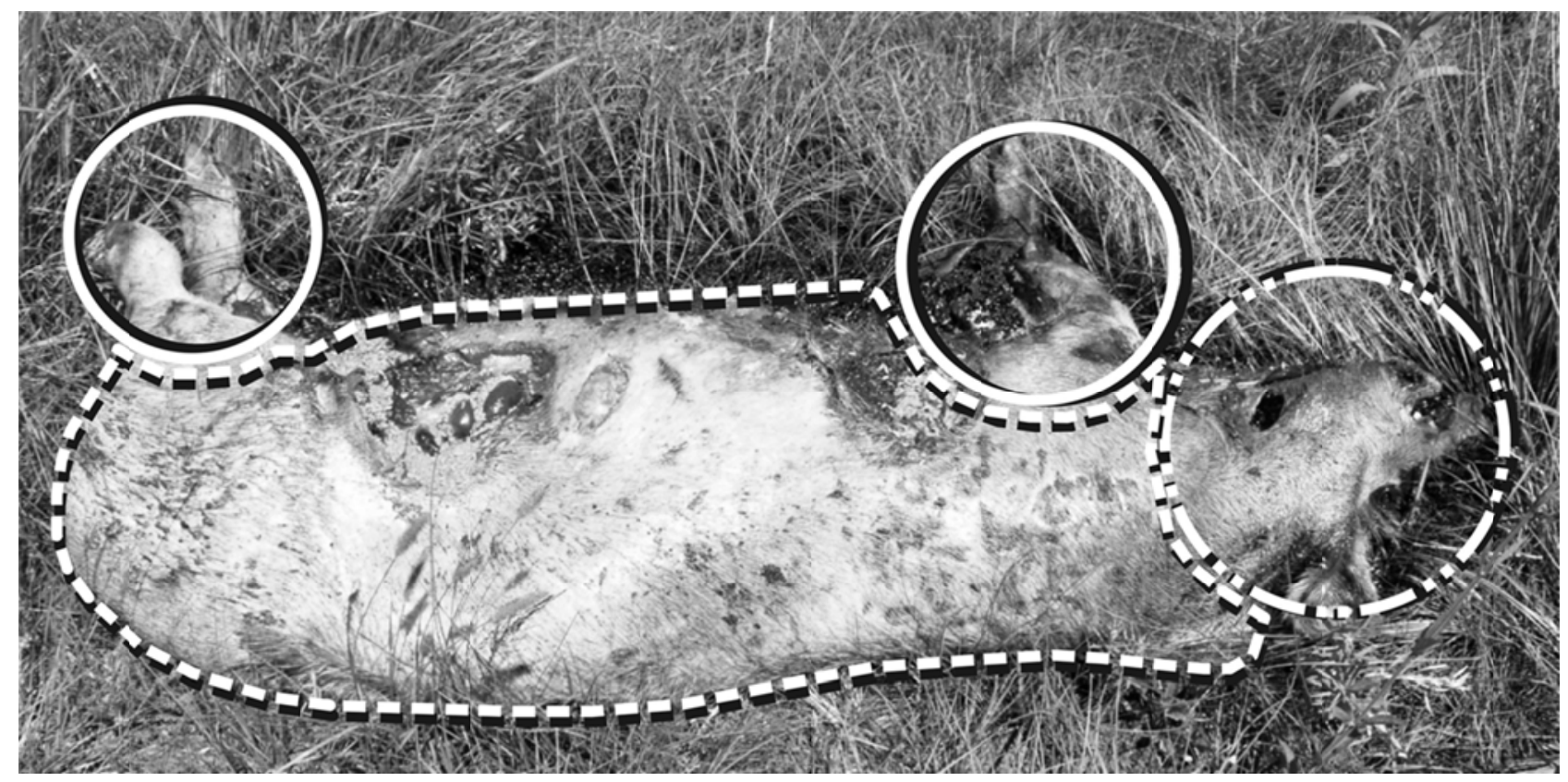

Fig. 1. An example of a pig in the advanced stage of decomposition (Head and neck $=7$, Trunk $=$

5, Limbs $=4$ for a combined score or TBS of 16). 


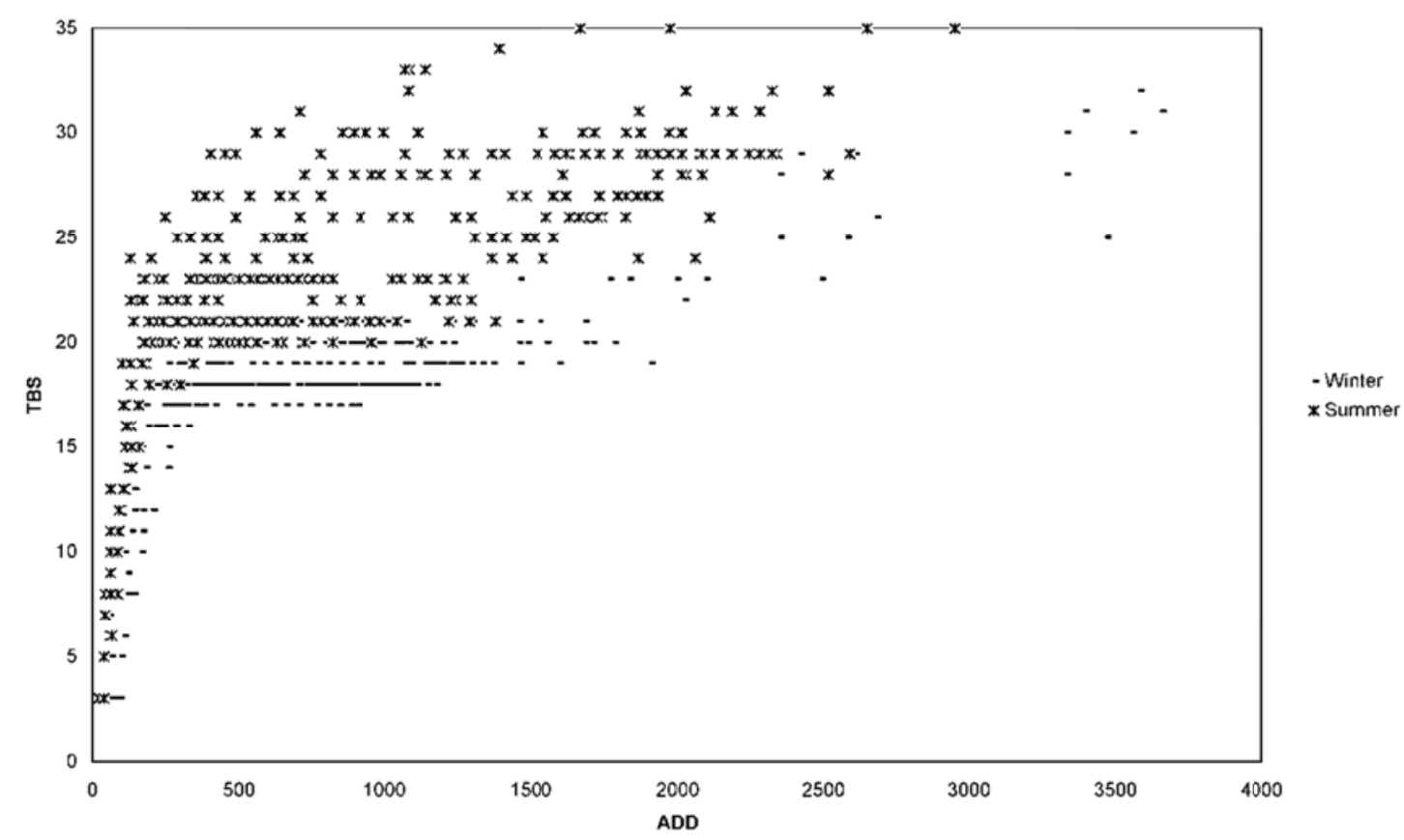

Fig. 2. ADD vs. TBS for the entire pig dataset indicating the difference in seasonal distribution between winter (-) and summer pigs (X). 